\title{
Burnt-out Metastatic Prostate Cancer
}

\author{
Dong Suk Shin, Dong Hoe Koo, Suhyeon Yoo, Deok Yun Ju, Cheol Min Jang, \\ Kwan Joong Joo', Hyun Chul Shin ${ }^{2}$, Seoung Wan Chae ${ }^{3}$ \\ Departments of Internal Medicine, ${ }^{1}$ Urology, ${ }^{2}$ Neurosurgery, ${ }^{3}$ Pathology, Kangbuk Samsung Hospital, \\ Sungkyunkwan University School of Medicine, Seoul, Korea
}

\begin{abstract}
A burnt-out prostate cancer tumor is a very rare clinical entity. The term 'burnt-out' refers to a primary tumor that has spontaneously and nearly completely regressed without treatment. Since metastasis of prostate cancer is usually encountered in the presence of advanced disease, distant metastasis with an undetectable primary tumor is very rare. We report herein a case of a burnt-out prostate cancer tumor that metastasized to the thoracic $(T)$ spine and caused cord compression. A 66-year-old man visited the Emergency Department due to weakness of both legs for the past two days. His blood and urine tests were normal at the time. His spine magnetic resonance imaging (MRI) scans looked like bone metastasis that involved the T-7 vertebral body and a posterior element, and caused spinal cord compression. Other images, including from the brain MRI, neck/chest/abdomino-pelvic computed tomography (CT) scan and 18F-fluorodeoxyglucose (FDG)-positron emission tomography (PET) and endoscopy, revealed no lesions that suggested malignancy. After total corpectomy T-7 and screw fixation/fusion at T5 to T10, the pathology report revealed a metastatic carcinoma that was strongly positive for prostate-specific antigen (PSA). The serum PSA value was $1.5 \mathrm{ng} / \mathrm{mL}$. The transrectal 12-core prostate biopsy and ultrasonography showed no definitive hypoechoic lesion, but one specimen had slight (only $1 \%$ ) adenocarcinoma with a Gleason score of $6(3+3)$. The final diagnosis was burned-out prostate cancer with an initial normal PSA value. Although metastatic disease with an unknown primary origin was confirmed, a more aggressive approach in seeking the primary origin could provide a more specific treatment strategy and greater clinical benefit to patients.
\end{abstract}

Key Words: Prostate cancer, Metastasis, Regression

\section{INTRODUCTION}

Prostate carcinoma has a well-known pattern of metastasis; it presents by metastases to the axial skeleton and local lymph nodes. ${ }^{1}$ Prostate-specific antigen (PSA) level was found to be high in $40-67 \%$ of localized and $76-100 \%$ of metastatic prostate cancer, ${ }^{2}$ and was significantly higher in patients with bone metastases. ${ }^{3}$

A burnt-out prostate cancer tumor is a very rare clinical

Received: June 3, 2013, Revised: June 26, 2013,

Accepted: July 2, 2013

Corresponding Author: Dong Hoe Koo, Department of Internal Medicine, Kangbuk Samsung Hospital, Sungkyunkwan University School of Medicine, 108, Pyung-Dong, Chongno-Gu, Seoul 110-746, Korea

Tel: 82-2-2001-2531, Fax: 82-2-2001-1596

E-mail: d.h.koo@samsung.com entity. The term 'burnt-out' refers to a primary tumor that has spontaneously and near completely regressed without treatment. ${ }^{4}$ Several cases of burnt-out germ cell tumors, such as testis cancer, have been reported, but reports of burnt-out metastatic prostatic cancer were not found in the literature. Therefore, we report a patient with burnt-out prostate cancer that metastasized to the spine causing cord compression.

\section{CASE}

A 66-year-old man visited the emergency department of our institution with weakness in both legs for the previous 2 days. The patient complained of increasing pain at the thoracic (T) spine 8-9 level over the previous 2 months. Physical examination revealed decreased sensation (numbness, pinprick) and motor power at both lower extremities, but intact voiding 
sensation and anal tone. And additional examination of other systems, including the respiratory and gastrointestinal systems, was normal.

The patient's complete blood count (CBC) and urine microscopy were in the normal range at that time. In addition, blood chemistry, including liver/kidney function and electrolytes, were within normal range, except for a slightly increased alkaline phosphatase (ALP, $133 \mathrm{IU} / \mathrm{L}$ ). Chest X-ray was also normal. Magnetic resonance imaging (MRI) scan of the spine look like bone metastasis involving the T-7 vertebral body and posterior element, causing spinal cord compression at the T-7 level (Fig. 1A). Further evaluations were performed in order to determine the primary origin. Tumor markers, including alpha-fetoprotein (AFP), $2.18 \mathrm{ng} / \mathrm{mL}$, carcinoembryonic antigen (CEA), $1.18 \mathrm{ng} / \mathrm{mL}$, carbohydrate antigen 19-9 (CA19-9), $9.00 \mathrm{ng} / \mathrm{mL}$, were all within the normal range. Also, the initial PSA value was within the normal range (1.48 ng/ $\mathrm{mL})$. Other images including brain MRI, neck/chest/abdominopelvic computed tomography (CT) scan, esophagogastroduodenoscopy, and colonoscopy revealed no suspicious lesions suggesting malignancy. In addition, 18F-fluorodeoxyglucose (FDG)-positron emission tomography (PET) scan revealed T-spine uptake, but there was no uptake in other areas (Fig. 1B). Needle biopsy was performed for the spinal mass, but just the atypical tumor cells in the bone marrow were reported (Fig. 2).

Although the patient's paraplegia was slightly improved after steroid injection during the hospital course, the patient decided to undergo surgery rather than radiotherapy because of the rapid progression of paraplegia and uncertainty of primary malignancy. After total corpectomy T-7 and screw fixation/fusion at the T-5 to T-10 vertebral body, the pathology report revealed the metastatic carcinoma with a strong positive for PSA (Fig 2B), and negative for cytokeratins 7 (CK7), CK20, thyroid transcription factor-1 (TTF-1), lung cancer alliance (LCA), cluster of differentiation 56 (CD56), S-100, and vimentin. Serum PSA value was $1.5 \mathrm{ng} / \mathrm{mL}$. The 12-core transrectal prostate biopsy and ultrasonography showed no definitive hypoechoic lesion, prostate volume of $28.4 \mathrm{gm}$. The pathology result reported that 11 of 12 tissue specimens revealed benign prostatic glands only, but one specimen had a little portion of adenocarcinoma ( $1 \%$ only), with a Gleason score of $6(3+3)$ (Fig. 3) and the result of prostatic biopsy correspond to the result of T-7 metastatic carcinoma patho-
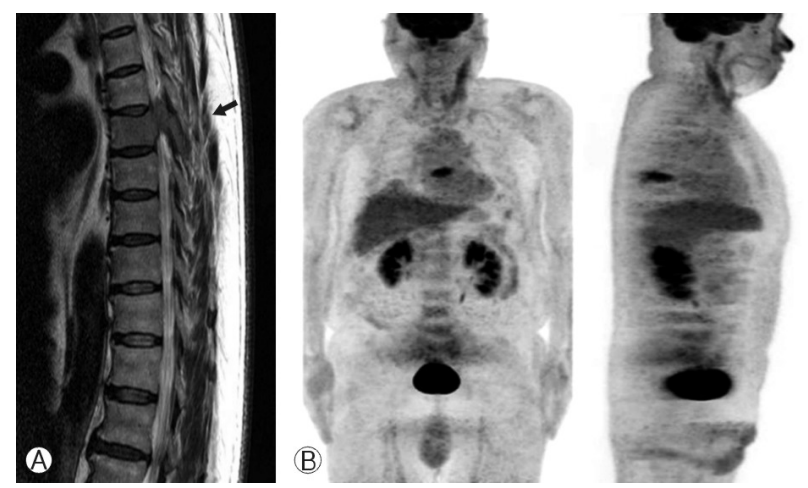

Fig. 1. Spine MRI and PET/CT findings. (A) Bone metastasis involving the T-7 vertebral body and posterior element (arrow), causing spinal cord compression on spine MRI. (B) Hypermetabolism in T-7 on PET/CT.

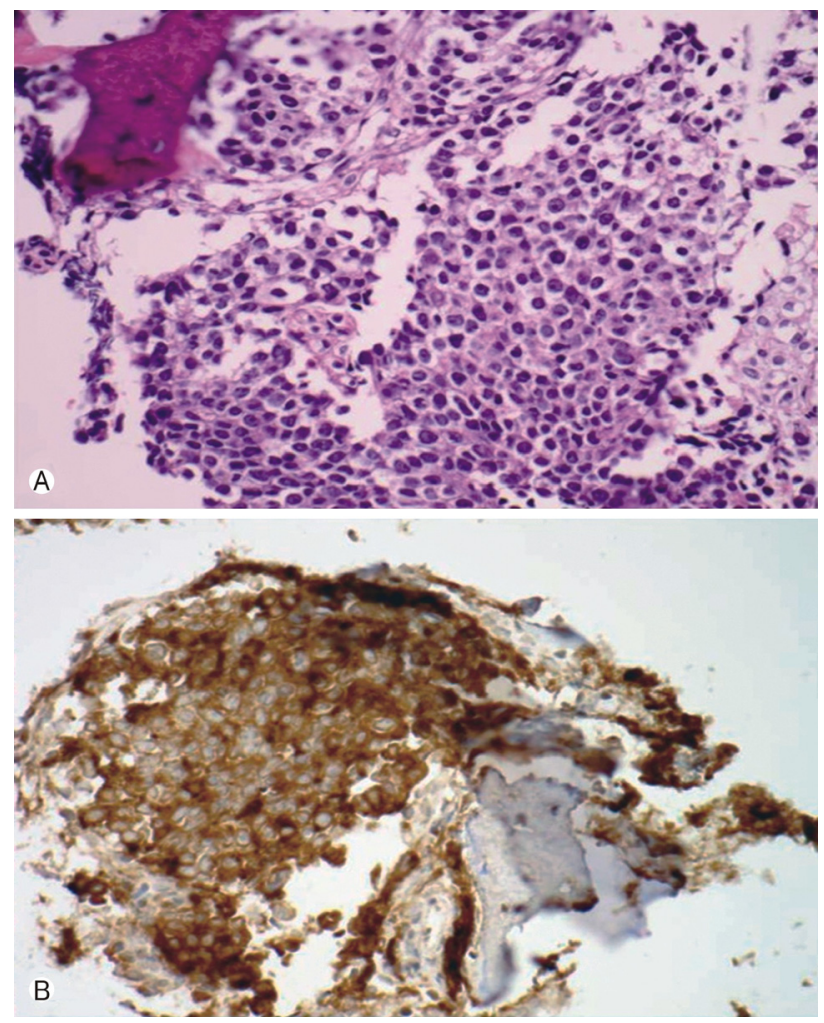

Fig. 2. Microscopic findings of T-7 vertebral mass. (A) The epithelioid tumor cells are arranged in solid sheets (H\&E stain, $\times 400)$. (B) The tumor cells are positive for PSA (Immunohistochemical stain, $\times 400)$.

logy. Although we checked serial PSA and free PSA, the results were still in the normal range; PSA $1.34 \mathrm{ng} / \mathrm{mL}$, free PSA $0.21 \mathrm{ng} / \mathrm{mL}$. The final diagnosis was burnt-out prostate cancer with an initial normal PSA value. Because the patient was considered to have no residual disease with a normal range of PSA, post-operative radiotherapy to the spine (total 


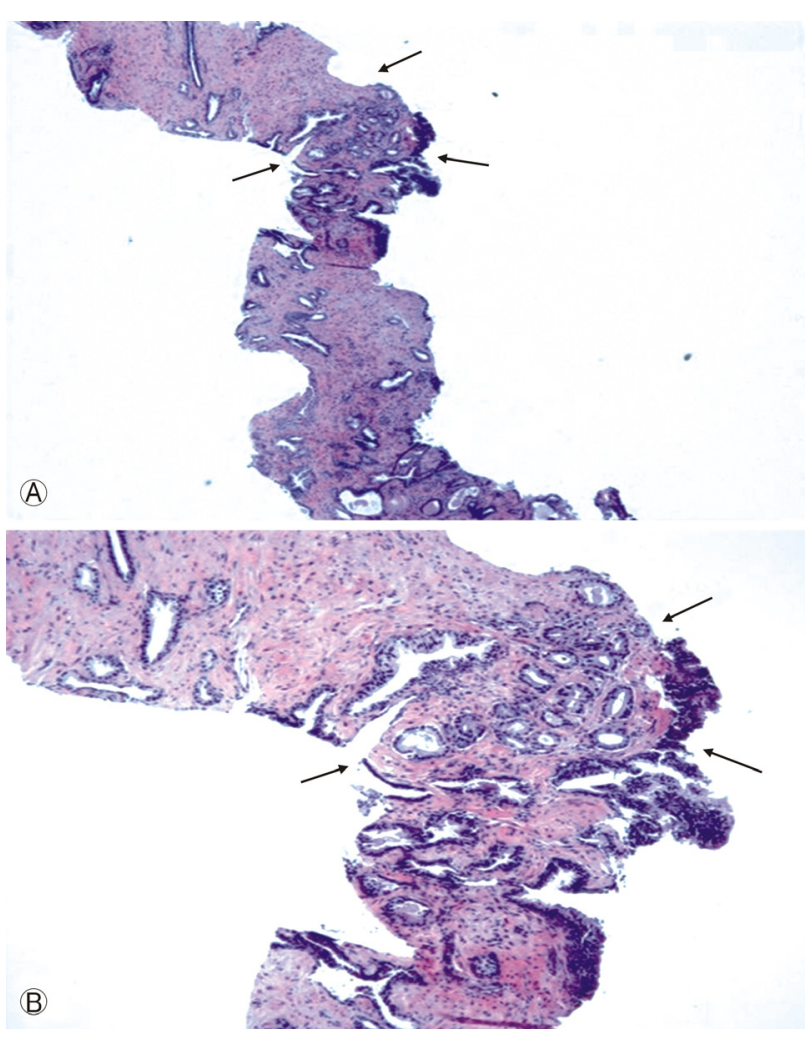

Fig. 3. Microscopic findings of prostate. (A) Low magnification shows adenocarcinoma area (arrows) (H\&E stain, $\times 40)$. (B) The medium-sized tumor glands (arrows) are present (H\&E stain, $\times 100)$.

$30 \mathrm{~Gy} / 10$ fraction) and active surveillance without anti-hormonal therapy were carried out. During a 3-month follow-up, the patient remained asymptomatic with a low level of PSA $(1.8 \mathrm{ng} / \mathrm{mL})$.

\section{DISCUSSION}

Prostate cancer is the most commonly diagnosed cancer, and the second most common cause of cancer-related death among men in the United States, exceeded only by lung cancer. ${ }^{5}$ Serum PSA, discovered in 1971, is considered the most important biomarker for detecting, staging, and monitoring cancer of the prostate in its early stage. ${ }^{6,7}$ Almost all patients with metastatic prostate cancer present with a high serum PSA level. ${ }^{8}$ Bone is the most common site for metastasis in prostate cancer patients. ${ }^{9}$ In a recent autopsy study of 1,589 patients with prostate cancer, $90 \%$ of patients with metastases had bony involvement, while only $10 \%$ of patients had only nonbony hematogenous metastases such as lung, liver, pleura and adrenal gland. ${ }^{1}$

However, metastatic prostate cancer with normal level of serum PSA has been reported in several studies. Yamamoto et al. reported M1 prostate cancer patients with low levels of PSA. ${ }^{10}$ Among the 167 patients with M1 prostate cancer, 8 patients had a serum PSA level of less than $10 \mathrm{ng} / \mathrm{mL}$, and mostly had poorly differentiated or undifferentiated carcinoma and relatively poor prognosis. Nishio et al. also reported that serum PSA did not aid in the follow-up of disease in patients who had initially low PSA levels, and CEA or chromogranin A (CgA) may be more helpful in follow-up. ${ }^{11}$ Leibovici et al. described characteristics of prostate cancer progression with a low PSA level. ${ }^{12}$ The reported characteristics were highgrade, locally advanced tumors, especially when atypical histologic variants are present. The phenomenon of prostate cancer progression without concomitant PSA elevation may be explained by the proliferation of cell lines that either cannot produce PSA or are poorly differentiated prostate cancer cells that have lost their ability to express PSA. ${ }^{13}$ Even if these patients with low PSA levels are diagnosed with advanced prostate cancer, tumors were usually found at the prostate as the primary origin.

Vanishing prostate cancer was first reported by DiGiuseppe et al., and its incidence was 2 cases among 3,038 patients. ${ }^{14}$ Vanishing prostate cancer was defined as a phenomenon in which a radical prostatectomy fails to show cancer that was diagnosed by needle biopsy of the prostate. Vanishing prostate cancer has been reported by several authors. ${ }^{15-19}$ Our case, however, did not undergo radical prostatectomy, and does not fit into the definition of vanishing prostate cancer. In addition, there have been some reports of burnt-out tumors which were found in metastatic lesions, but where the primary lesion could not be found. Most of these cases were associated with testis germ-cell tumors, and there have been no reports involving prostate cancer like our case.

Although our patient had an advanced metastatic disease with neurologic complications, the primary lesion was not initially detected, and the metastatic lesion was localized. Very low volume of tumor and intermediate Gleason score, indicating favorable histologic differentiation, usually suggest the very early stages of prostate cancer. If information about the spinal lesion had not been delivered to the pathologists, the burnt out prostate cancer might not have been found. Because we decided to treat with aggressive surgical manage- 
ment rather than conservative manage or palliative radiation therapy, the patient was able to get a definitive diagnosis and plan further.

Although our case presented as a metastatic disease of unknown primary origin, we diagnosed it as burnt-out prostate cancer with normal PSA level. Prostate cancer can present as an advanced disease with low serum PSA levels and may progress after burn-out at the primary site. Because this is an uncommon clinical situation, a more aggressive approach to determine the primary origin could provide a more specific treatment strategy and greater clinical benefit for patients.

\section{REFERENCES}

1. Bubendorf L, Schöpfer A, Wagner U, Sauter G, Moch H, Willi N, et al. Metastatic patterns of prostate cancer: an autopsy study of 1,589 patients. Hum Pathol 2000;31:578-83.

2. Chybowski FM, Keller JJ, Bergstralh EJ, Oesterling JE. Predicting radionuclide bone scan findings in patients with newly diagnosed, untreated prostate cancer: prostate specific antigen is superior to all other clinical parameters. J Urol 1991;145:313-8.

3. Wang ZL, Wang XF. Relationship of serum prostate-specific antigen and alkaline phosphatase levels with bone metastases in patients with prostate cancer. Zhonghua Nan Ke Xue 2005;11:825-7.

4. Lopez JI, Angulo JC. Burned-out tumour of the testis presenting as retroperitoneal choriocarcinoma. Int Urol Nephrol 1994;26:549-53.

5. Siegel R, Ward E, Brawley O, Jemal A. Cancer statistics, 2011: the impact of eliminating socioeconomic and racial disparities on premature cancer deaths. CA Cancer J Clin 2011;61:212-36.

6. Rao AR, Motiwala HG, Karim OM. The discovery of prostate-specific antigen. BJU Int 2008;101:5-10.

7. Polascik TJ, Oesterling JE, Partin AW. Prostate specific anti- gen: a decade of discovery--what we have learned and where we are going. J Urol 1999;162:293-306.

8. Stamey TA, Kabalin JN, Ferrari M, Yang N. Prostate specific antigen in the diagnosis and treatment of adenocarcinoma of the prostate. IV. Anti-androgen treated patients. J Urol 1989;141:1088-90.

9. Thobe MN, Clark RJ, Bainer RO, Prasad SM, RinkerSchaeffer CW. From prostate to bone: key players in prostate cancer bone metastasis. Cancers (Basel) 2011;3:478-93.

10. Yamamoto S, Ito T, Akiyama A, Aizawa T, Miki M, Tachibana M. M1 prostate cancer with a serum level of prostate-specific antigen less than $10 \mathrm{ng} / \mathrm{mL}$. Int J Urol 2001;8:374-9.

11. Nishio R, Furuya Y, Nagakawa O, Fuse H. Metastatic prostate cancer with normal level of serum prostate-specific antigen. Int Urol Nephrol 2003;35:189-92.

12. Leibovici D, Spiess PE, Agarwal PK, Tu SM, Pettaway CA, Hitzhusen K, et al. Prostate cancer progression in the presence of undetectable or low serum prostate-specific antigen level. Cancer 2007;109:198-204.

13. Lee DK, Park JH, Kim JH, Lee SJ, Jo MK, Gil MC, et al. Progression of prostate cancer despite an extremely low serum level of prostate-specific antigen. Korean J Urol 2010; 51:358-61.

14. DiGiuseppe JA, Sauvageot J, Epstein JI. Increasing incidence of minimal residual cancer in radical prostatectomy specimens. Am J Surg Pathol 1997;21:174-8.

15. Bostwick DG, Bostwick KC. 'Vanishing' prostate cancer in radical prostatectomy specimens: incidence and long-term follow-up in 38 cases. BJU Int 2004;94:57-8.

16. Moskaluk CA. Vanishing prostate cancer syndrome: symptom of a larger clinical issue. Am J Surg Pathol 2005;29:561-3.

17. Trpkov K, Gao Y, Hay R, Yimaz A. No residual cancer on radical prostatectomy after positive 10-core biopsy: incidence, biopsy findings, and DNA specimen identity analysis. Arch Pathol Lab Med 2006;130:811-6.

18. Ebel Sepúlveda L, Corti D, Foneron A, Troncoso L, Gil G, Carpio D, et al. Vanishing prostate cancer. clinical presentation and histological review. Actas Urol Esp 2009;33:956-9.

19. Lee SR, Ham WS, Kim WT, Ju HJ, Lee JS, Choi YD. Pathologic characteristics and prognosis of pathologic T0 prostate cancer. Korean J Urol 2009;50:229-36. Korean. 